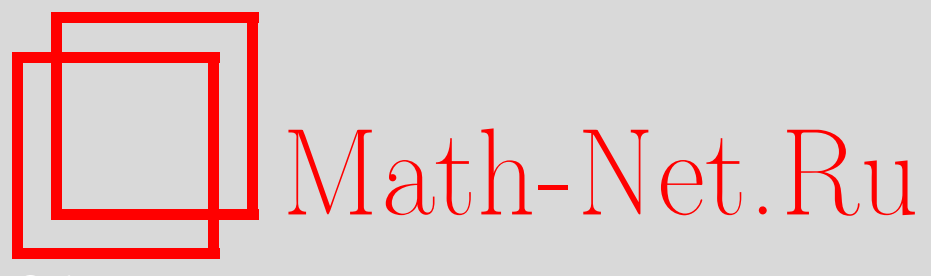

Л. М. Самойлов, Аналог теоремы Левицкого для бесконечно порожденных ассоциативных алгебр, Матем. заметки, 2009, том 86, выпуск 1, 151-153

DOI: https://doi.org/10.4213/mzm8363

Использование Общероссийского математического портала Math-Net.Ru подразумевает, что вы прочитали и согласны с пользовательским соглашением http: //www . mathnet.ru/rus/agreement

Параметры загрузки:

IP : 3.81 .55 .215

26 апреля 2023 г., 11:22:49

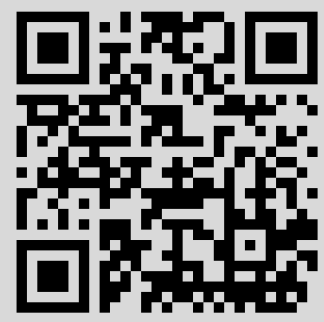




\section{Аналог теоремы Левицкого для бесконечно порожденных ассоциативных алгебр}

\section{Л. М. Самойлов}

Все рассматриваемые в работе алгебры являются ассоциативными алгебрами над полем.

Из теоремы Левицкого о положительном решении проблемы Куроша-Левицкого для PI-алгебр вытекает, что если PI-алгебра $A$ порождается множеством $\left\{a_{1}, \ldots, a_{k}\right\}$ и любое слово от множества образующих $\left\{a_{i}\right\}$ является нильпотентным степени не выше $m$, то сама алгебра $A$ является нильалгеброй ограниченного индекса $N$. Число $N$ зависит от степени тождества, $m$ и $k$. Доказательство этого утверждения и его обобщений, содержится, например, в [1] или [2].

В рассмотренном выше утверждении над полями нулевой характеристики убрать условие конечной порожденности нельзя, что показывает следующий простой пример.

Пример. Пусть $F$ - поле нулевой характеристики. Рассмотрим свободную ассоциативно-коммутативную алгебру $F\left[x_{1}, x_{2}, \ldots\right]$ над $F$ без единицы, порожденную счетным множеством $\left\{x_{1}, x_{2}, \ldots\right\}$. Пусть $A=F\left[x_{1}, x_{2}, \ldots\right] / I$, где идеал $I$ порожден всеми соотношениями $x_{i}^{2}=0$. Тогда в алгебре $A$ любое слово от образующих $a_{i}=x_{i}+I$ нильпотентно степени 2. Однако

$$
\left(a_{1}+\cdots+a_{n}\right)^{n}=n ! a_{1} a_{2} \cdots a_{n} \neq 0 .
$$

Таким образом, $A$ не является нильалгеброй ограниченного индекса.

Заметим, что если в примере поле $F$ имеет характеристику $p>0$, то $A$ - нильалгебра индекса $p$. Ниже мы докажем теорему 1 , которая показывает, что и в общем случае над полями положительной характеристики условие конечной порожденности в классической формулировке проблемы Куроша-Левицкого является лишним.

Теорема 1. Пусть $A$ - ассочиативная алгебра над полем характеристики $p>0$, удовлетворяющая тождеству $f=0$. Тогда если $A$ порождается множеством $\left\{a_{i}\right.$, $i \in I\}$, и любое слово от элементов $a_{i}$ нильпотентно степени не выше $m$, то $A$ является нильалгеброй ограниченного индекса $N$. При этом $N$ зависит от характеристики $p$, тождества $f$ и числа $m$ (и не зависит от мощности множества I).

ДоКАЗАТЕЛЬСтво теоремы 1 основывается на положительном решении проблемы Кемера о нильиндексе радикала относительно свободной ассоциативной алгебры [3], которое было получено автором в [4] для многообразий сложности $<p$ и в [5] в общем случае.

Теорема 2 [5]. Радикал относительно свободной ассоциативной алгебры счетного ранга над бесконечным полем положительной характеристики является нильидеалом ограниченного индекса.

ДокАЗАТЕЛЬСтво теоремы 1. Ясно, что достаточно доказать теорему 1 только для алгебр над бесконечными полями характеристики $p>0$. Поэтому будем считать, что $A$ - алгебра над $F, F$ - бесконечное поле характеристики $p>0$. Через $\operatorname{Var}(A)$ будем обозначать многообразие, порожденное алгеброй $A$. Обозначим через $F\langle X\rangle$ свободную ассоциативную алгебру (без единицы), порожденную счетным множеством $X$. Пусть $\Gamma \subseteq F\langle X\rangle$ - идеал тождеств алгебры $A$. Тогда $F\langle X\rangle / \Gamma$ - относительно свободная алгебра счетного ранга в многообразии $\operatorname{Var}(A)$. Напомним, что сложностью $T$-идеала $\Gamma$

Работа выполнена при поддержке Российского фонда фундаментальных исследований (грант № 07-01-00080).

(C) Л. М. САмойлов, 2009 
(а также сложностью соответствующего ему многообразия $\operatorname{Var}(A))$ называется наибольшее число $n$ со свойством $\Gamma \subseteq T\left[M_{n}\right]$, где $T\left[M_{n}\right]$ - идеал тождеств алгебры матриц $M_{n}$ порядка $n$. Сложность многообразия конечна по теореме Амицура-Левицкого, за исключением многообразия, состоящего только из нулевой алгебры. Хорошо известно, что радикал алгебры $F\langle X\rangle / \Gamma$ совпадает с $T\left[M_{n}\right] / \Gamma$.

Будем вести индукцию по сложности многообразия $\operatorname{Var}(A)$. Для многообразий сложности 0 теорема очевидна, так как каждое такое многообразие удовлетворяет тождеству $x^{N}=0$ для некоторого $N$.

Пусть сложность многообразия $\operatorname{Var}(A)$ равна $n$. Рассмотрим идеал $I$ алгебры $A$, порожденный множеством

$$
\left\{h\left(a_{1}, \ldots, a_{s}\right) \mid a_{i} \in A, h \in T\left[M_{n}\right]\right\} .
$$

Из теоремы 2 вытекает, что $I$ - нильидеал ограниченного индекса. Поэтому достаточно доказать, что факторалгебра $B=A / I$ будет нильалгеброй ограниченного индекса. Через $M$ обозначим образ множества всех слов над алфавитом $\left\{a_{i}, i \in I\right\}$ при естественном отображении $A \rightarrow A / I$.

Алгебра $B$ удовлетворяет всем тождествам $M_{n}$. Хорошо известно, что $M_{n}$ обладает полилинейным центральным полиномом $g\left(x_{1}, \ldots, x_{l}\right)$ (см., например, [6]), причем любой центральный полином будет тождеством алгебры $M_{n-1}$. Рассмотрим множество $J$, состоящее из линейных комбинаций элементов $b_{0} g\left(b_{1}, \ldots, b_{l}\right), b_{i} \in M$. Ясно, что $J$ является двусторонним идеалом алгебры $B$.

Так как полином $g$ полилинеен, алгебра $B / J$ удовлетворяет тождеству

$$
x_{0} g\left(x_{1}, \ldots, x_{l}\right)=0,
$$

поэтому сложность многообразия $\operatorname{Var}(B / J)$ не превосходит $n-1$. Следовательно, по предположению индукции $B / J$ будет нильалгеброй ограниченного индекса. Для доказательства теоремы 1 достаточно показать, что идеал $J$ также является нильалгеброй ограниченного индекса.

Рассмотрим произвольный элемент $b \in J$. Тогда $b$ имеет вид $b=\sum \alpha_{i} u_{i}, \alpha_{i} \in F$, где каждый из элементов $u_{i}$ можно представить в виде $u_{i}=b_{0}^{(i)} g\left(b_{1}^{(i)}, \ldots, b_{l}^{(i)}\right), b_{j}^{(i)} \in M$. Тогда элемент $b^{N}=\left(\sum \alpha_{i} u_{i}\right)^{N}$ является линейной комбинацией элементов $L_{N, \rho}\left(u_{i_{1}}, \ldots, u_{i_{t}}\right)$, где через $L_{N, \rho}\left(x_{1}, \ldots, x_{t}\right)$ обозначена частичная линеаризация полинома $x^{N}$ мультистепени $(\rho)=\left(\rho_{1}, \ldots, \rho_{t}\right)$ по переменным $\left(x_{1}, \ldots, x_{t}\right)$. Учитывая, что значения полинома $g$ лежат в центре алгебры $B$, получаем

$$
L_{N, \rho}\left(u_{i_{1}}, \ldots, u_{i_{t}}\right)\left(g\left(b_{1}^{\left(i_{1}\right)}, \ldots, b_{l}^{\left(i_{1}\right)}\right)\right)^{\rho_{1}} \cdots\left(g\left(b_{1}^{\left(i_{t}\right)}, \ldots, b_{l}^{\left(i_{t}\right)}\right)\right)^{\rho_{t}} L_{N, \rho}\left(b_{0}^{\left(i_{1}\right)}, \ldots, b_{0}^{\left(i_{t}\right)}\right) .
$$

В работе [3] Кемером было показано, что существует такая константа $q$, что следствиями тождества $f=0$ являются все частичные линеаризации $L_{N, \rho}$ тождества $x^{N}=0$ при достаточно больших $N$, имеющие степень $<N / q$ по каждой из переменных. Далее рассмотрим два случая.

1) Для всех $\rho_{i}$ выполнено $\rho_{i}<N / q$. Тогда при достаточно больших $N$

$$
L_{N, \rho}\left(b_{0}^{\left(i_{1}\right)}, \ldots, b_{0}^{\left(i_{t}\right)}\right)=0,
$$

поэтому элемент $(*)$ равен нулю в алгебре $B$; следовательно, и в идеале $J$.

2) Для некоторого $i$ (пусть для $i=1$ ) выполнено $\rho_{i} \geqslant N / q$. Заметим, что элемент $g\left(b_{1}^{\left(i_{1}\right)}, \ldots, b_{l}^{\left(i_{1}\right)}\right)$ является линейной комбинацией не более чем $l$ ! слагаемъх, каждое из которых лежит в $M$. Пусть это будет линейная комбинация мономов $v_{1}, \ldots, v_{l^{\prime}}$, $l^{\prime} \leqslant l$ !. По теореме А.И. Ширшова о высоте, примененной к $l$-порожденной подалгебре $\left\langle v_{1}, \ldots, v_{l^{\prime}}, 0, \ldots, 0\right\rangle$ алгебры $B$, при достаточно больших $N / q(N / q$ зависит от $l$, но не от элементов $\left.v_{1}, \ldots, v_{l^{\prime}}\right)$ элемент $g\left(b_{1}^{\left(i_{1}\right)}, \ldots, b_{l}^{\left(i_{1}\right)}\right)^{\rho_{1}}$ является линейной комбинацией таких 
слов в алфавите $v_{1}, \ldots, v_{l^{\prime}}$, каждое из которых содержит $m$-ю степень некоторого подслова. Но $m$-я степень любого слова из $M$ равна нулю. Таким образом, в рассматриваемом случае при достаточно больших $N$ элемент $(*)$ тоже равен нулю. При этом $N$ не зависит от $b$ и мощности множества $I$.

Итак, при достаточно больших $N$, не зависящих от числа порождающих, элемент $b^{N}=\left(\sum \alpha_{i} u_{i}\right)^{N}$ равен линейной комбинации нулевых слагаемых, т.е. он равен нулю. Теорема 1 доказана.

\section{СПИСОК ЦИТИРОВАННОЙ ЛИТЕРАТУРЫ}

[1] E. Zelmanov, Nil Rings and Periodic Groups, KMS Lecture Notes Math., Korean Math. Soc., Seoul, 1992. [2] A. Ya. Belov, V. V. Borisenko, V. N. Latyshev, J. Math. Sci. (N. Y.), 87:3 (1997), 3463-3575. [3] A. Kemer, Trends in Ring Theory (Miskolc, Hungary, 1996), CMS Conf. Proc., 22, Amer. Math. Soc., Providence, RI, 1998, 59-69. [4] Л. М. Самойлов, Матем. заметки, 82:4 (2007), 583-592. [5] Л. М. Самойлов, Матем. сб., 199:5 (2008), 81-126. [6] Ю. П. Размыслов, Тождества алгебр и их представлений, Наука, М., 1989.

Л. М. Самойлов

Поступило

Ульяновский государственный университет

16.09.2008

E-mail: samoilov_l@rambler.ru 